\title{
Exercise and education for community- dwelling older participants with knee osteoarthritis: a video-linked programme protocol based on a randomised controlled trial
}

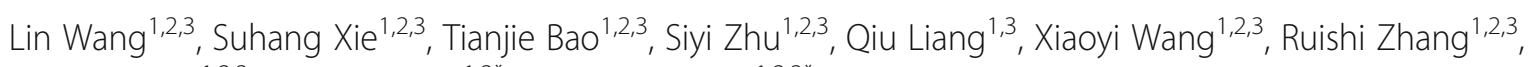
Xiaona Xiang ${ }^{1,2,3}$, Chunping Du ${ }^{1,3^{*}}$ and Chengqi He ${ }^{1,2,3^{*}}$

\begin{abstract}
Background: Neuromuscular and quadriceps exercises have been shown to be effective approaches to relieve pain and to improve function for patients with knee osteoarthritis. In this study, we aim to provide an informative feasible model in which therapeutic exercise and education will be undertaken with physiotherapy supervision and instruction via video link. We also aim to explore the relationship between program-induced pain alleviation/ functional improvements and reduction in irritability, which might be mediated through program-induced psychosocial benefits.

Methods: In this proposed two-parallel group (neuromuscular exercise versus quadriceps exercise), single-blinded, randomised controlled trial, participants aged $\geq 50$ years with osteoarthritic knee pain will undergo a 12-week intervention, comprising video-linked education, supervised exercises, and a 12-week follow-up. Seven measurements will be taken to collect longitudinal data. A generalised estimating equation will be used to establish the adjusted difference in effectiveness on pain, function, irritability, and psychosocial outcomes between participants undertaking neuromuscular exercises and those undertaking quadriceps exercises. The primary outcomes are overall average pain in the knee joint during walking, as assessed through the 11-point Numerical Pain Rating Scale, and the Western Ontario and McMaster Universities osteoarthritis index physical function subscale. Furthermore, pressure pain threshold and changes in self-report pain scores pre-, during, and post-exercise were also measured as an indication of irritability. In addition, both the 6-min walk test and a timed up \& go test were used to assess walking function performance. Finally, patients' emotions (e.g., fear and catastrophising), self-trust, needs in terms of disease knowledge, mental resilience, social support and health-related quality of life were investigated. Two four-wave cross-lagged models will be used to investigate directional relationships, aiming to investigate the complex mechanisms concerning the effects of exercise programmes.
\end{abstract}

*Correspondence: ducp118@163.com; hxkfhcq2015@126.com

'Department of Rehabilitation Medicine, West China Hospital, Sichuan University, No. 37 Guoxue Xiang, Chengdu, Sichuan 610041, P. R. China

Full list of author information is available at the end of the article

(c) The Author(s). 2021 Open Access This article is licensed under a Creative Commons Attribution 4.0 International License, which permits use, sharing, adaptation, distribution and reproduction in any medium or format, as long as you give appropriate credit to the original author(s) and the source, provide a link to the Creative Commons licence, and indicate if changes were made. The images or other third party material in this article are included in the article's Creative Commons licence, unless indicated otherwise in a credit line to the material. If material is not included in the article's Creative Commons licence and your intended use is not permitted by statutory regulation or exceeds the permitted use, you will need to obtain permission directly from the copyright holder. To view a copy of this licence, visit http://creativecommons.org/licenses/by/4.0/ The Creative Commons Public Domain Dedication waiver (http://creativecommons.org/publicdomain/zero/1.0/) applies to the data made available in this article, unless otherwise stated in a credit line to the data. 
Discussion: Through summarising the study's strengths and limitations, this study may provide promising insights in terms of exercise therapy optimisation for people with knee osteoarthritis and/or other chronic pain within a psychosocial framework.

Trial registration: ChiCTR2100041978 (chictr.org.cn), January 10, 2021.

Keywords: Community-dwelling older adults, Knee osteoarthritis, Exercise, Education, Irritability, Psychosocial mechanism

\section{Background}

Knee osteoarthritis (KOA) was reported to have involved living a global total of 8.3 million years with a disability between 1997 and 2017 [1], and 4.15 million years lived with a disability between 2012 and 2018 in China [2]. With global ageing and an increased prevalence in obesity [3], a continued rise is anticipated. Clinical practice guidelines strongly recommend education and individualised exercise as a first-line option for managing KOA symptoms $[4,5]$.

Neuromuscular exercise, based on biomechanics and neuromuscular control principles, comprises a series of motions or actions aimed at improving sensorimotor control and achieving functional stability [6], in which there is an emphasis on the quality of open and closed chain movements through performing functionally weight-bearing exercises with correct posture and alignment [7]. Quadriceps exercises aim to improve muscular mass, strength, and endurance through undertaking a range of reduced weightbearing open-chain exercises to limit direct pressure on the knee, to strengthen the major muscle groups, and to improve joint stability [8].

Recent clinical trials have suggested that both exercise regimens could markedly ease pain and improve function [9-11]; however, whether significant differences in pain-relieving and functional improvement effects exist between the two exercise types is controversial. There is, however, limited evidence supporting the difference in effects between these two types of exercise when they are combined with education and when they are delivered using mobile online programs and physiotherapy supervision [12].

Peripheral and central pain sensitisation is considered to be the cause of KOA-related pain, the features of which are associated with self-reported pain severity [13, 14]. In quantitative sensory testing, pain sensitisation is often expressed as a pressure pain threshold (PPT) and temporal summation due to mechanical stimulation [15]; whereas, in clinical pain management, physiotherapists focus on irritability [16]. According to Maitland's definition, the more severe the pain is in response to a given physical activity or mechanical stimulation, the more irritable the pain becomes. Irritability can be assessed as the intensity of activity required to cause pain, severity of induced pain, and duration of pain [17].

Pain sensitivity during a given physical activity has been reported to be an independent predictor of activity performance and pain-related impairment in communal participation for patients with KOA [16]. A recent trial that measured the trajectory of pain during 12 weeks of neuromuscular training reported that changes in pain during training, induced through progressive exercise, decreased over time and reached a plateau in the 10th week [18]. Moreover, it has been shown that exerciseinduced analgesia is characterised as reduced sensitivity to painful stimuli post-training [19]. As indicated, assessing irritability and the long- or short-term effects of exercise programs in relieving pain could be relevant [20].

Psychological effects of exercise include acquiring illness knowledge, reducing helplessness, increasing self-efficacy (a person's self-trust in performing certain goal-oriented tasks [21]), optimising coping strategies (the efforts to deal with and minimise the effects of illness [22]), strengthening social support, and relieving depression [23]. Moreover, KOA symptoms, especially chronic pain and functional restrictions [3, 24], affect patient psychosocial status. Exactly, exercise has benefits within psychosocial domains that vary with the effects of controlled symptoms or irritability, rather than remaining static and unchanging characteristics $[23,25]$. Changes in mental characteristics could be in reaction to analgesic and functional benefits and other behaviours involved in self-management. Selfefficacy, strengthened through KOA-specific exercise, has been reported to be an independent predictor of better functional performance [26].

In studying the association between self-efficacy and physiological efficacy, the assessment factors of psychological outcomes should be distinguished as static characteristics [27] or as a dynamically assessed progress during the exercise $[26,28]$, which conforms to the selfefficacy theory of Bandura [21, 29]. Besides, three out of ten women with knee osteoarthritis tend to suffer from depression [30], which could be reduced by exercise programs [31, 32]. However, as with other psychological benefits, the relationship with patient-perceived relieved pain and improved function has not yet been established. 
A previous study by our research group indicated that Internet-based rehabilitation programs could improve pain but not physical function in patients with KOA [33]. In another unpublished study, we suggested that exercise effectively improved some domains of psychological health and that the management of pain and disability could be enhanced by improved psychological status (Study registration: PROSPERO 2021 CRD42021217822).

In the present study, we propose an informative feasible model of exercise for KOA. Specifically, the model involves physical therapists delivering remote video guidance for exercise and demand-oriented disease education online, as well as family support. This model may be beneficial to patients with KOA as a means to improve pain, physical function, mental health and social support. Given that a decrease in irritation could play a vital role in the improvement of pain and function [15, 17]. Psychosocial factors may also play an intermediary role in the process [23].

\section{Objective}

This clinical trial will investigate effects on pain, function, mental health, social participation/support, and health-related quality of life for community-dwelling older participants with $\mathrm{KOA}$ through a 12-week program that combines progressive exercise and systematic education under the video-linked instruction of a physiotherapist and with the support of a participant's family. The key mechanisms of irritability, physiological outcomes, mental health, and social participation/support will be further explored. We hypothesised that: (i) progressive exercise and systematic education will benefit participants with $\mathrm{KOA}$, and that neuromuscular exercise will have a better effect than quadriceps exercise; (ii) pain relief benefits from a decrease in peripheral hyperalgesia after a period of regular exercise training, which is mediated through the improvement of mental health and social participation/support, and; (iii) after a period of regular exercise training, a decrease in exerciserelated irritability will enhance functional improvement, which is mediated through improvement in mental health and social participation/support.

\section{Trial design}

The design of this trial, involving video-linked instruction by physiotherapists and support from a participant's family in relation to education combing neuromuscular exercise versus quadriceps exercise for community-dwelling older participants with KOA, is a two-armed, randomised, positive-controlled, investigator-blinded, prospective and longitudinal study with a 12-week intervention along with a further 12-week follow-up period.

\section{Methods}

This protocol is guided by the Standard Protocol Items: Recommendations for Interventional Trials statement [34]. A flow diagram explaining the protocol timeline is shown in Fig. 1. The Ethics Committee on Biomedical Research, West China Hospital of Sichuan University has approved this trial (identifier: 2020-945).

\section{Study setting}

This trial will be conducted among community-dwelling older participants with KOA in Chengdu, Sichuan Province from December 2020 to December 2021. The intervention and evaluation site will be a participant's home or community.

\section{Participants}

Community-dwelling older adults are potentially eligible if they: (i) are aged $\geq 50$ years at the time of enrolment; (ii) meet the criteria for symptomatic KOA according to the American College of Rheumatology [35]; (iii) have a plain knee radiographic image indicating KOA grades 0III, using the Kellgren \& Lawrence atlas [36]; (iv) have a plain knee radiographic image showing joint space narrowing (grades 0-2), based on the Osteoarthritis Research Society International classification [36]; and (v) have the support of family members during every exercise session to ensure basic safety, the use of a video connection, and video-viewing facilities.

In relation to potential participants, exclusion criteria comprise: (i) having undergone arthroplasty, arthroscopy, or open surgery on the knee in the past 12 months, or planning to undergo an elective knee joint replacement surgery within the next 6 months; (ii) having other joint pathologies (for example, rheumatoid arthritis, severe osteoporosis, or fracture); (iii) having confirmed or suspected diseases that may restrict exercise (for example, cerebral haemangioma, exertional angina pectoris, severe anaemia, fixed-rate pacemaker, or complete atrioventricular block); (iv) being at risk of participating in exercise without supervision or screening while using the physical activity readiness questionnaire [37]; (v) having hypertension and diabetes mellitus without monitoring and treatment; (vi) having cognitive impairment as screened using Mini-Cog [38]; or (vii) having confirmed serious psychiatric disorders (for example, schizophrenia, major depression, paranoid psychosis, mania, or mental disorders due to mental retardation).

\section{Intervention}

The 12-week intervention will comprise exercise and education sessions conducted by four certified physiotherapists experienced in managing chronic musculoskeletal pain (QL, XW, RZ, and TW). Exercise training 


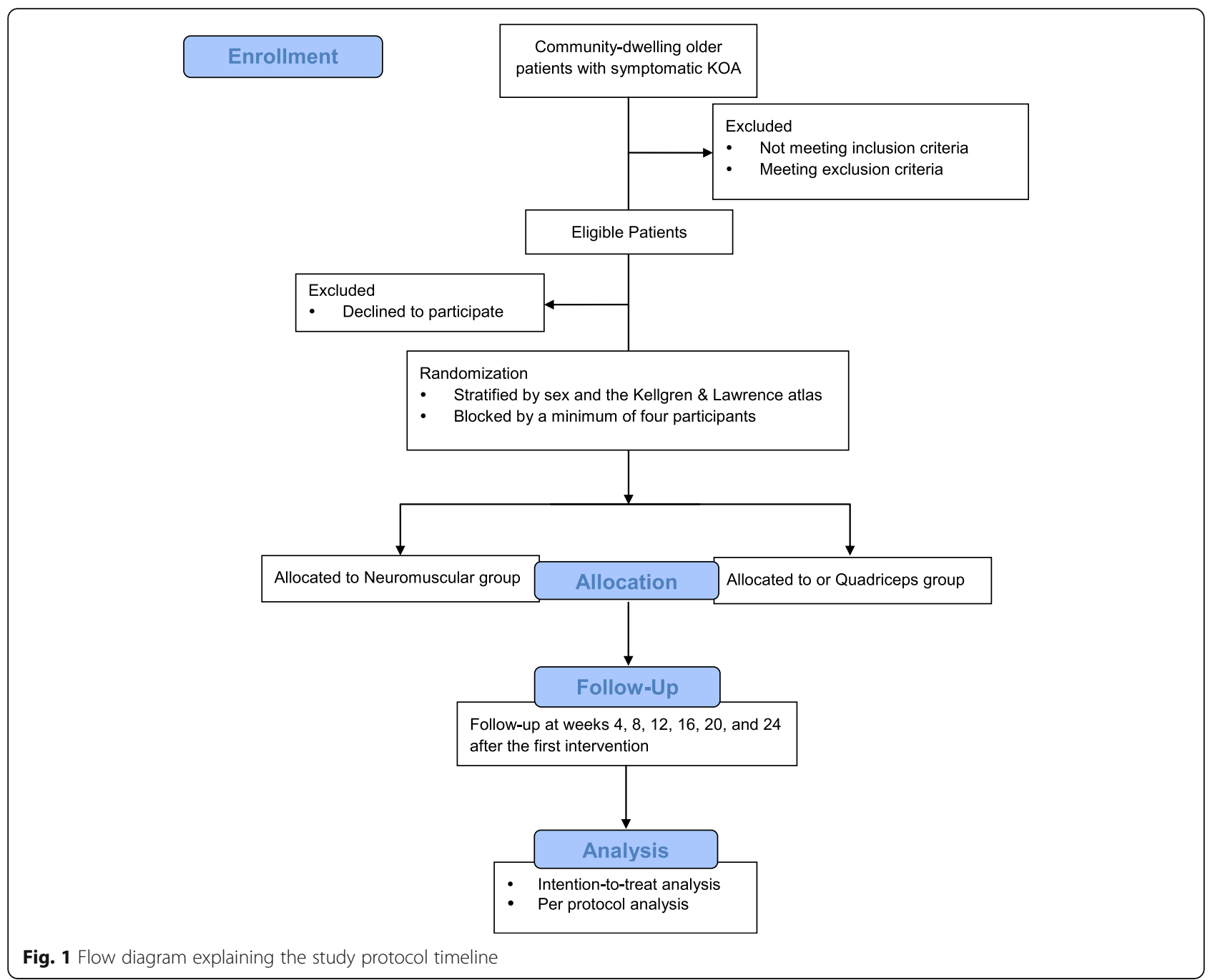

programs will differ between two groups, that is, one group performing neuromuscular exercise versus another group performing quadriceps exercise, with education based on individual needs following the same design between the two groups. Physiotherapists will attend a 4-h standardised training before the formal start of the trial, to ensure reliability and repeatability of the delivered interventions (both education and exercise). With the implementation of the proposal, a technical partner $(\mathrm{BC})$ will concurrently maintain and improve the functionality of a mobile online platform, namely, Joint Consultation, which has been developed to remind participants and deliver videos of specific home-based exercises, after which exercise diaries will be completed. In brief, reminders will be sent electronically concerning the video links and self-exercise for participants to accept the exercise prescription, to view videos of the exercises (also on the online platform), and to complete the exercise diary on Joint Consultation. Furthermore, a free, open-source software program (Tencent Meeting) will be used for video links between participants and physiotherapists.

\section{Exercise}

During the program, a participant will conduct three 30-40 min exercise sessions per week. A physiotherapist will supervise participants and deliver progressive exercise through a video connection weekly (12 sessions in total), and participants will exercise at home twice a week according to the exercise prescription (24 sessions in total). Family members will accompany the participants during each exercise session (including during video connection with the physiotherapist) to ensure safety and to provide support.

Our quadriceps exercise program will include nonweight-bearing exercises aimed to improve knee joint stability through enhancing endurance and the strength of the major muscle groups [8]. Our neuromuscular exercise program will include functional weight-bearing exercises 
aimed to promote functional stability along with alignment through improving sensorimotor control [6].

The messages twice a week used to remind to exercise before exercise at home, messages weekly used to remind to video-link with a physiotherapist, and exercise diary three times a week after every exercise session will be delivered to the participants with Joint Consultation. The results filling in the exercise diary feedback to the physiotherapist and clinical research assistant will be used as the record of participant attendance at-home exercise and feedback on symptom responses for calculating and monitoring adherence. Besides, the weekly video-linked session with the physiotherapist ensures the problems related to exercise and symptom responses can be informed and addressed in time, which could be considered as another action to monitor potential dropout situations or the loss of follow-up, and reduce the number of subjects who violate the allocation plan.

The exercise testing plan and exercise prescription are based on findings from previous studies [39-41]. In detail, the neuromuscular exercises will progress in difficulty monthly, and the quadriceps exercises will progress in resistance and in the number of sets weekly from the third training, which will be determined according to a participant's pain (defined as an average pain score $\geq 4$, on an 11-point numerical pain rating scale (NPRS) [42] during exercise) and perceived exertion (defined as an average exertion between 5 and 8, on an 11-point modified rating perceived exertion scale [43] during exercise).

\section{Education}

Participant needs-based education lasting 15-20 min will be conducted by the physiotherapist monthly through a video link (four sessions in total). The educational needs assessment tool (ENAT) [44] will be used to determine a participant's level of interest in terms of KOA knowledge, according to which individualised education will be planned and delivered. The education content corresponding to 39 ENAT items will be guided by clinical guidelines and previous evidence, and will mainly include information on the current condition and possible outcomes for each participant, the vital role of exercise and healthy lifestyle (e.g. weight control), options and methods to help alleviate symptoms, and other content intended to facilitate participants' understanding [4, 5, 45-53]. Moreover, the physiotherapists will encourage participants to review the education materials and will respond to any participants' questions [49].

\section{Outcome measures}

Table 1 summarises the primary and secondary outcome measures. Longitudinal evaluation will be conducted seven times in total over a 6-month period. As shown in the participant timeline (Table 2), outcome data will be collected at baseline, and at 4,8 , and 12 weeks after allocation, mostly comprising self-reported scales. Over a 3month follow-up, evaluations will be conducted at 4,8 , and 12 weeks post-intervention.

\section{Primary outcomes}

Primary outcomes are as follows: (i) overall average pain in the knee joint during walking over the previous month: an 11-point NPRS will be used to investigate participant-reported pain ranging from 0 (no pain) to 10 (the worst pain), with 1.8 units having been reported as a minimum clinically important difference (MCID) [54]; and (ii) difficulty with physical function: the Western Ontario and McMaster Universities osteoarthritis index (WOMAC) physical function subscale [55] will be used to evaluate self-reported dysfunction using 17 questions. Total subscale scores will range from 0 to 68 , with higher scores indicating more serious dysfunction. An MCID in terms of physical function in KOA has been determined as six units [56].

\section{Secondary outcomes}

Secondary outcomes are as follows:

(1) Knee pain over the previous $48 \mathrm{~h}$, determined using the WOMAC pain subscale [55] to evaluate selfreported pain in response to five questions. Total subscale scores will range from 0 to 20 , with higher scores indicating more serious pain.

(2) Irritability, with PPT assessed using a handheld pressure algometer (Wagner Force Ten, FDX 25, Greenwich) to reflect peripheral and central sensitisation in a resting state. PPT is calculated through an averaging of three repeat measurements at every assessment site, namely, the upper trapezius, biceps brachii, extensor carpi radialis longus, first dorsal interosseous, rectus femoris, vastus lateralis, and tibialis anterior muscles, and the medial compartment of the knee [20,57]. A 1 $\mathrm{cm}^{2}$ rubber tip press at a rate of $0.5 \mathrm{~kg} / \mathrm{s}$ on the centre of the site will be used and the number recorded when pressure sensations first change to slight pain. Data concerning changes in selfreported pain via an 11-point NPRS will be extracted from exercise diaries indicating exercisedependent peripheral sensitisation, where participants will be asked to self-report their pain scores pre- and post-exercise, and indicate their highest pain score during exercise [18].

(3) Functional physical performance, determined using a 6-min walk test and a timed up \& go (TUG) test to assess walking function performance [58]. 
Table 1 A summary of the outcome measures

\begin{tabular}{|c|c|c|c|}
\hline Measurements & Metric of measurement & Method of measurement & $\begin{array}{l}\text { Time point of } \\
\text { primary interest }\end{array}$ \\
\hline \multicolumn{4}{|l|}{ Primary outcomes } \\
\hline Self-reported pain & $\begin{array}{l}0-10 \text { (higher scores indicate more serious } \\
\text { pain) }\end{array}$ & 11-point numerical pain rating scale & \multirow{2}{*}{$\begin{array}{l}\text { Baseline, and } 4,8,12, \\
16,20 \text {, and } 24 \text { weeks } \\
\text { after allocation }\end{array}$} \\
\hline $\begin{array}{l}\text { Self-reported } \\
\text { function }\end{array}$ & $\begin{array}{l}\text { 0-68 (higher scores indicate more serious } \\
\text { dysfunction) }\end{array}$ & $\begin{array}{l}\text { Western Ontario and McMaster Universities } \\
\text { osteoarthritis index (WOMAC) - physical function } \\
\text { subscale }\end{array}$ & \\
\hline \multicolumn{4}{|l|}{ Secondary outcomes } \\
\hline Self-reported pain & $\begin{array}{l}0-20 \text { (higher scores indicate more serious } \\
\text { pain) }\end{array}$ & WOMAC pain subscale & \multirow{13}{*}{$\begin{array}{l}\text { Baseline, and } 4,8,12, \\
16,20 \text {, and } 24 \text { weeks } \\
\text { after allocation }\end{array}$} \\
\hline \multirow[t]{2}{*}{$\begin{array}{l}\text { Functional } \\
\text { performance }\end{array}$} & $\begin{array}{l}\text { Metres (longer distance indicates better } \\
\text { function) }\end{array}$ & 6-min walk test (6MWT) & \\
\hline & $\begin{array}{l}\text { Seconds (shorter time indicates better } \\
\text { function) }\end{array}$ & Timed up \& go (TUG) test & \\
\hline $\begin{array}{l}\text { Sensitisation in } \\
\text { resting state }\end{array}$ & $\begin{array}{l}\text { Kilogram (lighter pressure indicates a greater } \\
\text { degree of sensitisation or increased pain } \\
\text { sensitivity) }\end{array}$ & Pressure pain threshold (PPT) & \\
\hline $\begin{array}{l}\text { Exercise-dependent } \\
\text { sensitisation }\end{array}$ & $\begin{array}{l}0-11 \text { (higher scores indicate a greater degree } \\
\text { of sensitisation or increased pain sensitivity) }\end{array}$ & $\begin{array}{l}\text { Changes in self-reported pain pre-, post- and dur- } \\
\text { ing exercise via } 11 \text {-point numerical pain rating } \\
\text { scale in exercise diaries }\end{array}$ & \\
\hline Catastrophising & $\begin{array}{l}0-52 \text { (higher scores indicate a greater degree } \\
\text { of pain catastrophising) }\end{array}$ & Pain catastrophising scale (PCS) & \\
\hline $\begin{array}{l}\text { Fear related to } \\
\text { movements }\end{array}$ & $\begin{array}{l}\text { 17-68 (higher scores indicate more serious } \\
\text { fear) }\end{array}$ & Tampa scale for kinesiophobia (TSK) & \\
\hline Self-trust in exercise & $\begin{array}{l}0-70 \text { (higher scores indicate stronger } \\
\text { confidence) }\end{array}$ & Self-efficacy for exercise scale (SEES) & \\
\hline Self-trust in pain & $\begin{array}{l}0-50 \text { (higher scores indicate stronger } \\
\text { confidence) }\end{array}$ & Pain self-efficacy questionnaire (PSEQ) & \\
\hline $\begin{array}{l}\text { Acquisition of } \\
\text { knowledge about } \\
\text { KOA }\end{array}$ & $\begin{array}{l}0-156 \text { (higher scores indicate less knowledge } \\
\text { of KOA) }\end{array}$ & Educational needs assessment tool (ENAT) & \\
\hline Mental resilience & $\begin{array}{l}\text { 10-50 (higher scores indicate stronger } \\
\text { adaptation competence in the face of } \\
\text { problems) }\end{array}$ & $\begin{array}{l}\text { 10-item Connor-Davidson resilience scale (CD- } \\
\text { RISC-10) }\end{array}$ & \\
\hline $\begin{array}{l}\text { Perceived social } \\
\text { support }\end{array}$ & $\begin{array}{l}\text { 8-50 (higher scores indicate more satisfying } \\
\text { social support) }\end{array}$ & Satisfaction with received social support (SRSS) & \\
\hline $\begin{array}{l}\text { Pain affecting social } \\
\text { participation }\end{array}$ & $\begin{array}{l}\text { 16-96 (higher scores indicate more serious } \\
\text { impairment in social participation) }\end{array}$ & $\begin{array}{l}\text { Pain inference subscale of the West Haven Yale } \\
\text { multidimensional pain inventory (WHYMPI) }\end{array}$ & \\
\hline Quality of life & $\begin{array}{l}\text { Standardised scores for every section (higher } \\
\text { scores indicate more serious impairment in } \\
\text { social participation) }\end{array}$ & 36-item short-form health survey (SF-36) & $\begin{array}{l}\text { Baseline, and } 12 \text { and } \\
24 \text { weeks after } \\
\text { allocation }\end{array}$ \\
\hline \multicolumn{4}{|l|}{ Other assessments } \\
\hline $\begin{array}{l}\text { Comorbid } \\
\text { conditions }\end{array}$ & $\begin{array}{l}\text { 1-41 (higher scores indicate more complex } \\
\text { and dangerous comorbid conditions) }\end{array}$ & Charlson comorbidity index (CCI) & \multirow[t]{4}{*}{ Baseline } \\
\hline $\begin{array}{l}\text { Level of physical } \\
\text { activity }\end{array}$ & $\begin{array}{l}\text { Four levels divided into inactive, light-, } \\
\text { moderate-, hard-, and very hard-intensity. }\end{array}$ & Stanford brief activity survey (SBAS) & \\
\hline $\begin{array}{l}\text { Overall mental } \\
\text { health }\end{array}$ & $\begin{array}{l}\text { 14-70 (higher scores indicate better } \\
\text { psychological health) }\end{array}$ & $\begin{array}{l}\text { Warwick-Edinburgh mental well-being scale } \\
\text { (WEMWBS) }\end{array}$ & \\
\hline $\begin{array}{l}\text { Drugs, physical } \\
\text { therapies, and other } \\
\text { therapies used }\end{array}$ & Type, frequency, duration & Self-report & \\
\hline
\end{tabular}

Abbreviations: CCI Charlson comorbidity index, CD-RISC-10 10-item Connor-Davidson resilience scale, ENAT Educational needs assessment tool, KOA Knee osteoarthritis, PCS Pain catastrophising scale, PPT Pressure pain threshold, PSEQ Pain self-efficacy questionnaire, SBAS Stanford brief activity survey, SF-36 36-item short form health survey, SEES Self-efficacy for exercise scale, SRSS Satisfaction with received social support, TSK Tampa scale for kinesiophobia, TUG Timed up and go test, WEMWBS Warwick-Edinburgh mental well-being scale, WHYMPI West Haven Yale multidimensional pain inventory, 6MWT Six-minute walk test 


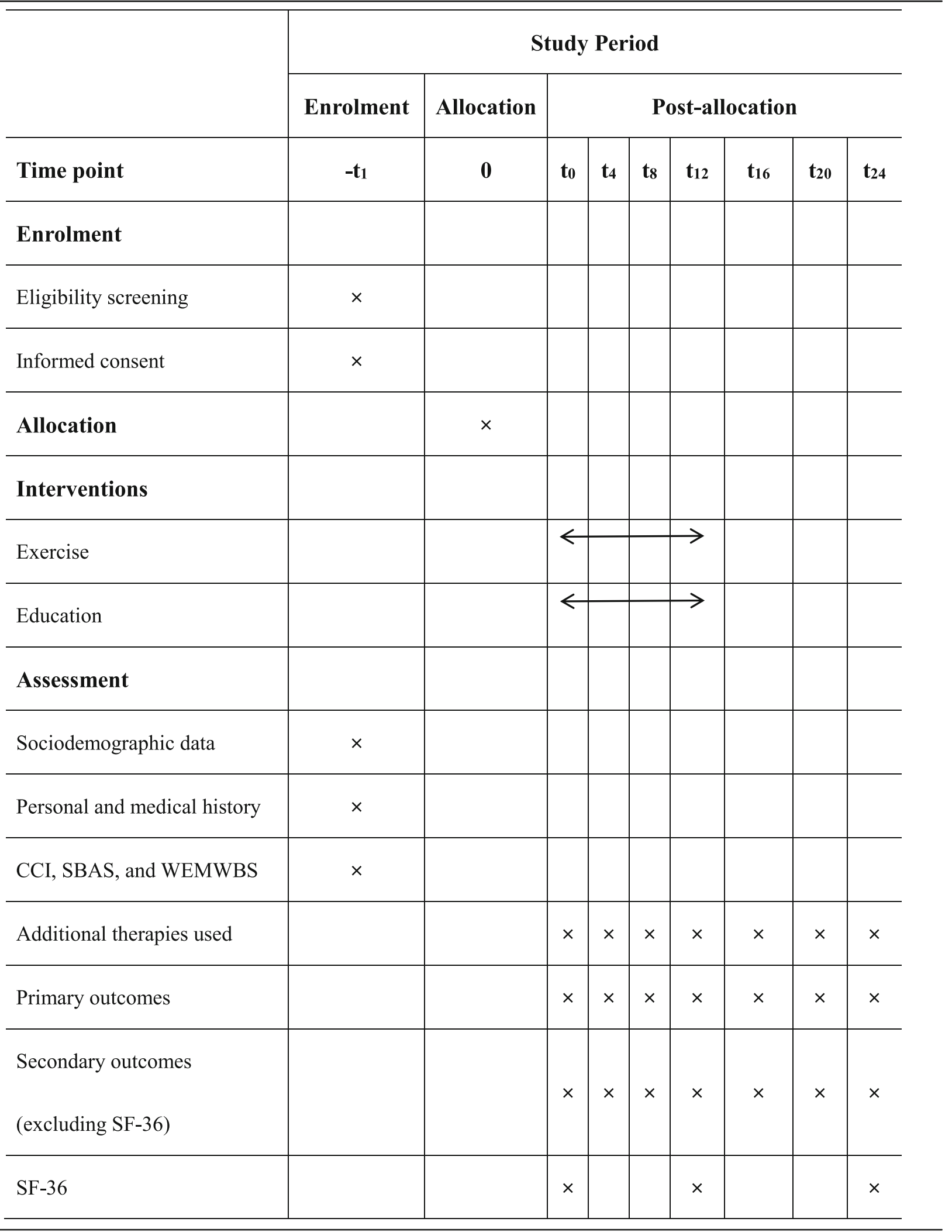


(4) Outcomes of mental health over the previous month, determined using the pain catastrophising scale [59], Tampa scale for kinesiophobia [60], selfefficacy for exercise scale [61], pain self-efficacy questionnaire, ENAT [44], and 10-item ConnorDavidson resilience scale [62] through evaluating the psychological status of the participants during exercise and follow-up, in terms of emotion (fear, catastrophising), self-trust, needs in terms of disease knowledge, and mental resilience domains.

(5) Outcomes of social participation and social support over the previous month, determined using the pain interference subscale of the West Haven Yale multidimensional pain inventory [63], and satisfaction with received social support questionnaire $[64,65]$ through evaluating the psychological status of the participants during exercise and follow-up, in terms of the effects of KOA symptoms and perceived support from family, community, and society.

(6) Outcomes of quality of life over the previous 3 months, determined in relation to health-related quality of life using the 36-item short-form health survey [66].

For baseline measures, the Charlson comorbidity index will be calculated to determine participants' comordibities [67]. The Stanford brief activity survey will be used to assess participants' baseline physical activity levels [68]. The Warwick-Edinburgh mental well-being scale will be used to assess participants' overall mental health [69]. To determine any additional therapies used by a participant during the intervention and follow-up period, we will record drug types and dosage, physical therapy, and any other therapies.

\section{Sample size}

PASS 15.0 (East Kaysville, USA) software will be used to test for two means in a repeated measures design, based on linear-mixed effect models [70-72], with estimations of the correlation coefficient between different observations made on the same participant set at 0.6 [9]. The sample size required will be sufficient to show a moderate effect size, that is, a 0.5 standard deviation in the betweengroup difference in relation to the primary outcomes (knee pain during walking and WOMAC function). With a yielding power of $80 \%$ and a significance level of 0.05 , the sample size is intended to comprise 44 participants in each group. With allowance for a dropout rate of $20 \%$, each group requires 55 enrolled participants.

\section{Recruitment}

Dissemination of recruitment information will be transmitted by facilities or institutions providing multiple services within a certain region, including sub-district offices, primary and secondary schools (for students' grandparents), colleges for senior students, activity centres or day-care centres for older adults in the community, and community medical institutions. Contact details or addresses concerning these facilities or institutions will be obtained from the Chengdu Civil Affairs Bureau (cdmzj.chengdu.gov.cn), Chengdu Education Bureau (edu.chengdu.gov.cn), and Chengdu Health Committee (cdwjw.chengdu.gov.cn), and these units will be invited to disseminate recruitment information onand offline. Interested older adults will be able to voluntarily enrol online. Eligible participants will receive electronic material concerning information necessary for participation, complete the online questionnaires, and upload their signatures, with participants able to contact the research team via phone to ask questions or to consult with them.

\section{Participant assignment for the intervention}

Eligible participants will be randomly assigned to either a neuromuscular group or a quadriceps group at a ratio of 1:1. Eligible participants will be stratified according to sex and the Kellgren \& Lawrence atlas, and allocation will be computer-generated in varied blocks involving a minimum of four participants, according to the enrolment sequence per week, using the R Software 3.6.1 ( $R$ Foundation for Statistical Computing, Vienna, Austria) statistical package. Randomisation will be conducted by an independent analyst (YK) at the clinical medical school who is not involved in the study or the intervention. Participants and physiotherapists will be kept informed through online messages. Considering the nature of the intervention, an informed allocation is essential for the participants and interventionists in the study; however, evaluators, data managers, and statistical analysts will be blinded.

\section{Data collection and management}

All outcome data will be collected by three specific evaluators (HT, ZZ, and KZ) at participants' homes or communities and will be stored electronically. An electronic database has been developed to manage clinical data in our department [73]. Exercise diaries will be stored after every session or home-based exercise in the database attached to Joint Consolation, from which data concerning exercise-dependent changes in pain will be extracted. Our evaluators will enter the data, and data verification, mainly in relation to missing and inconsistent data, will be performed by an independent medical school graduate (LX).

\section{Statistical methods}

We will mainly follow the intention-to-treat principle. A per-protocol analysis will be conducted among those 
who complete 8 of 12 in-person video-link sessions and 16 of 24 home exercises, according to allocation.

For continuous data, quantile-quantile plots will be constructed to determine the standardised residuals and histograms with normal distribution curves. For descriptive statistics, continuous data with normal distributions, continuous data with non-normal distributions, and categorical data will be described using means \pm standard deviations, medians (interquartile ranges), and frequencies, respectively. The significance level will be set at 0.05 for all analyses, and a two-sided 95\% confidence interval will be used.

A generalised estimating equation (GEE) will be used to test the hypothesis comparing between-group differences in terms of effects on primary and secondary outcomes. Covariates and possible confounders will be adjusted, including sociodemographic variables, baseline values of the outcome variables, stratification factors (grade of Kellgren \& Lawrence atlas), and noncomparable variables at baseline [74]. Additionally, we will identify other underlying predictors from the general KOA status and review treatments that accompany our intervention or follow-up, which will be selected $(p<$ 0.2 ) according to a univariate analysis using a backward stepwise procedure. Furthermore, for ordinal categorical secondary outcomes, we will fit the GEE using an alternating logistic regression model, assuming an exchangeable correlation structure, adjusted for all the predictors, and with final results reported as odds ratios. Given a close relation to the quasi-likelihood resolution of GEE [75], maximum likelihood estimation will be used to impute missing data.

To test the hypothesis concerning the mechanisms associated with the effects on pain and function, a cross-lagged model fitted within the structural equation modelling (SEM) will be used. SEM is an approach that allows simultaneous modelling of several variables and enables an investigation of more complex mediation models [76-78]. The variables of interest as mediators in the relationship between effects on pain/function and irritability are changes in outcomes on mental health and on social participation/support, which will be studied using two separate four-wave cross-lagged path models (during the intervention and follow-up). The time lag between each measurement will be 4 weeks. In addition to estimating cross-lagged effects, the specified model will include correlations within time points, autoregressive effects (stability), and underlying predictors, similar to the process of GEE analysis [79]. Moreover, full-information maximum likelihood will be used to handle missing data and estimate covariance matrices [80]. Additionally, data from both groups will be included in the cross-lagged model coded as two classified labels.

\section{Monitoring \\ Data monitoring \\ Not applicable.}

\section{Harms}

Any negative health events occurring during the trial (from enrolment to the last follow-up) will be considered as adverse events, regardless of the causal relationship with the intervention. Adverse events will be identified through three mechanisms: (i) supervised care and support from the family, who will be required to accompany a participant during three exercise sessions per week (including the video-linked session with the physiotherapist); (ii) participants who report the absence of supporting family for whom the medical institution in the community will be contacted to provide supervision; and (iii) participants being required to report any adverse events to the clinical coordinator (KS) by phone or to the physiotherapist via video link. A reported adverse event will be discussed by four doctors (HG, QW, RA, and YL) at our department, and suggestions will be processed within the community medical institution or within our department, according to the suggestions, as necessary.

\section{Auditing \\ Not applicable.}

\section{Discussion}

Considering the important role of supervision and instruction from physiotherapists in exercise therapy as well as safety during exercise and access and availability to mobile devices, we have designed and proposed to verify an intervention model that combines video-linked sessions instructed by a physiotherapist and support from family members. We proposed in this study three hypotheses to be verified in future research. Furthermore, the benefits of the informative feasible model of Internet-based exercise guidance for patients with KOA could be illustrated and, to some extent, the underlying mechanisms of the benefits could be explored.

Our study has some strengths. First, we will design an individualised exercise and education program for community-dwelling older participants. We will use online video-linked instruction and a mobile online platform to deliver the intervention, and the process will be supported by family members. ENAT results will help to guide the systematic education provided, which is intended to help achieve needs-based education.

Second, perceived psychosocial variables, such as potential mediators, will be included and assessed, thus providing greater depth to the study's interpretation of effects in relation to pain reduction and functional improvement [76, 77]. Psychological benefits of exercise for patients with knee osteoarthritis have been well- 
documented by recent studies [31, 32]. However, there is a knowledge gap to bridge in the relationship between the improved interested psychological domains and the ameliorated results of pain and function before and after the exercise intervention, instead of considering the psychological assessments as a baseline or as characteristics of the participants [81-83].

Third, longitudinal data from this randomised trial using a GEE will allow us to draw conclusions with a high level of external validity [84] and will help identify the most effective form of physiotherapist-instructed and family-supported exercise through the mobile online platform. The conclusions of this study are likely to help development of an evidence-based online exercise program to manage the symptoms and mental status of patients with KOA, which will also allow optimisation of exercise therapy from a psychosocial view $[4,5,85,86]$.

However, some potential limitations also exist in the proposed study. First, self-reported heterogeneous symptoms of KOA cover a wide range of pain severity, disability, and personal experience (to address this wide range, we will assess and control psychological resilience) $[87,88]$. Second, we will include variables related to changes in osteoarthritic pain during the exercise sessions based on findings from previous studies that may not have considered all the variables involved in the multidimensional nature of pain [78]. Third, the intervention in this study involved the support from the participants' families, which only will be recorded as the number of accompanied exercise sessions by monthly survey, but not details of family support and companionship shown. Although, the details could be questioned in the interview after the period of the intervention, the evidence-based quantitative tools to assess this factor could be a future direction of study. Finally, as with all cross-lagged models, our findings concerning the mechanisms for pain are exploratory and require external validation [76-78].

\section{Study status}

Seventy-one participants were enrolled in the study as of April 21, 2021. The enrolment of the first participant was on February 23, 2021.

\section{Abbreviations}

ENAT: Educational needs assessment tool; GEE: Generalised estimating equation; KOA: Knee osteoarthritis; MCID: Minimum clinically important difference; NPRS: Numerical pain rating scale; PPT: Pressure pain threshold; SEM: Structural equation modelling; WOMAC: Western Ontario and McMaster Universities osteoarthritis index

\section{Acknowledgements}

We thank Professor Xing Zhao for his support of statistics. We respect and appreciate the developers and verifiers of the scales and/or tools mentioned in the study.

\section{Confidentiality}

Anonymisation will be conducted through numbering each participant according to the sequence of enrolment in the electronic database, which will be completed before statistical analysis by an independent analyst. Any exported personal data for the specific task will be pseudonymised.

\section{Authors' contributions}

$\mathrm{CH}$ and $\mathrm{LW}$ designed the trial. LW, TB, XW, RZ, and QL designed the education and exercise intervention. SZ, SX, CD, and XX developed the mobile platform (Joint Consultation). LW designed the statistical analysis. LW, TB, and SX drafted the manuscript. All authors met the Authorship Criteria as set by the International Committee of Medical Journal Editors. The author(s) read and approved the final manuscript.

\section{Funding}

The study is supported by the National Natural Science Foundation (81972146 to Chengqi He), the Department of Science and Technology of Sichuan Province (2020YJ0210 to Chengqi He), and the Nursing Discipline Development Special Fund Project of West China Hospital, Sichuan University (HXHL20001 to Chengqi He). External peer-review took place during the funding process. All the funders play no role in the design, later conduction, or reporting of this study.

Availability of data and materials

Enquired in advance, the corresponding author will provide the datasets of the study for rational purposes.

\section{Declarations}

\section{Ethics approval and consent to participate}

The Ethics Committee on Biomedical Research, West China Hospital of Sichuan University has approved this trial (identifier: 2020-945). Any modifications to the protocol will be represented on Chinese Clinical Trial Registry (chictr.org.cn). Each participant in this study will obtain a written informed consent before the allocation. Before baseline assessment, Wejuanxing (a platform of the online survey) will be used for collecting informed consent electronically, while the handwritten informed consent will be collected at the baseline assessment. Moreover, the participant and the main family supporter will sign the informed consent file.

\section{Consent for publication}

Not applicable.

\section{Competing interests}

None.

\section{Author details}

'Department of Rehabilitation Medicine, West China Hospital, Sichuan University, No. 37 Guoxue Xiang, Chengdu, Sichuan 610041, P. R. China. ${ }^{2}$ Key Laboratory of Rehabilitation Medicine, West China Hospital, Sichuan University, Chengdu, Sichuan 610041, P. R. China. ${ }^{3}$ Institute of Rehabilitation Medicine, West China Hospital, Sichuan University, Chengdu, Sichuan 610041, P. R. China.

Received: 7 March 2021 Accepted: 5 May 2021

Published online: 22 May 2021

\section{References}

1. Khan G, Fitzmaurice C, Naghavi M, Ahmed LA. Global and regional incidence, mortality and disability-adjusted life-years for Epstein-Barr virusattributable malignancies, 1990-2017. BMJ Open. 2020;10(8):e037505. https:// doi.org/10.1136/bmjopen-2020-037505.

2. Liu Q, Wang S, Lin J, Zhang Y. The burden for knee osteoarthritis among Chinese elderly: estimates from a nationally representative study. Osteoarthr Cartil. 2018;26(12):1636-42. https://doi.org/10.1016/j.joca.2018.07.019.

3. Hunter DJ, Bierma-Zeinstra S. Osteoarthritis. Lancet (London, England). 2019; 393:1745-59. https://doi.org/10.1016/s0140-6736(19)30417-9.

4. Bannuru RR, Osani MC, Vaysbrot EE, Arden NK, Bennell K, Bierma-Zeinstra SMA, et al. OARSI guidelines for the non-surgical management of knee, hip, and polyarticular osteoarthritis. Osteoarthr Cartil. 2019:27(11):1578-89. https://doi.org/10.1016/j.joca.2019.06.011. 
5. Kolasinski SL, Neogi T, Hochberg MC, Oatis C, Guyatt G, Block J, et al. 2019 American College of Rheumatology/Arthritis Foundation guideline for the management of osteoarthritis of the hand, hip, and knee. Arthritis Care Res. 2020;72(2):149-62. https://doi.org/10.1002/acr.24131.

6. Ageberg E, Nilsdotter A, Kosek E, Roos EM. Effects of neuromuscular training (NEMEX-TJR) on patient-reported outcomes and physical function in severe primary hip or knee osteoarthritis: a controlled before-and-after study. BMC Musculoskelet Disord. 2013;14(1):232. https://doi.org/10.1186/1471-24 74-14-232.

7. Ageberg E, Roos EM. Neuromuscular exercise as treatment of degenerative knee disease. Exerc Sport Sci Rev. 2015;43(1):14-22. https://doi.org/10.1249/ jes.0000000000000030

8. Brosseau L, Taki J, Desjardins B, Thevenot O, Fransen M, Wells GA, et al. The Ottawa panel clinical practice guidelines for the management of knee osteoarthritis. Part two: strengthening exercise programs. Clin Rehabil. 2017; 31(5):596-611. https://doi.org/10.1177/0269215517691084.

9. Bennell KL, Kyriakides M, Metcalf B, Egerton T, Wrigley TV, Hodges PW, et al. Neuromuscular versus quadriceps strengthening exercise in patients with medial knee osteoarthritis and varus malalignment: a randomized controlled trial. Arthritis Rheumatol. 2014;66(4):950-9. https://doi.org/10.1 002/art.38317.

10. Rashid SA, Moiz JA, Sharma S, Raza S, Rashid SM, Hussain ME. Comparisons of neuromuscular training versus quadriceps training on gait and WOMAC index in patients with knee osteoarthritis and Varus Malalignment. J Chiropr Med. 2019;18(1):1-8. https://doi.org/10.1016/j.jcm.2018.07.003.

11. Qiu L, Chengqi H, Junliang J, Haolun Y, Lin Y. The effects of neuromuscular exercise programme on pain and physical function in patients with knee osteoarthritis. West China Med J. 2018;33:1247-51. https://doi.org/10.7507/1 002-0179.201805116

12. Schäfer AGM, Zalpour C, von Piekartz H, Hall TM, Paelke V. The efficacy of electronic health-supported home exercise interventions for patients with osteoarthritis of the knee: systematic review. J Med Internet Res. 2018;20(4): e152. https://doi.org/10.2196/jmir.9465.

13. Arendt-Nielsen L, Nie H, Laursen MB, Laursen BS, Madeleine P, Simonsen $\mathrm{OH}$, et al. Sensitization in patients with painful knee osteoarthritis. Pain. 2010;149(3):573-81. https://doi.org/10.1016/j.pain.2010.04.003.

14. King CD, Sibille KT, Goodin BR, Cruz-Almeida Y, Glover TL, Bartley E, et al. Experimental pain sensitivity differs as a function of clinical pain severity in symptomatic knee osteoarthritis. Osteoarthr Cartil. 2013;21(9):1243-52. https://doi.org/10.1016/j.joca.2013.05.015.

15. Neogi T, Frey-Law L, Scholz J, Niu J, Arendt-Nielsen L, Woolf C, et al. Sensitivity and sensitisation in relation to pain severity in knee osteoarthritis: trait or state? Ann Rheum Dis. 2015;74(4):682-8. https://doi.org/10.1136/a nnrheumdis-2013-204191.

16. Wideman $\mathrm{TH}$, Edwards RR, Finan PH, Haythornthwaite JA, Smith MT. Comparing the predictive value of task performance and task-specific sensitivity during physical function testing among people with knee osteoarthritis. J Orthop Sports Phys Ther. 2016;46(5):346-56. https://doi.org/1 0.2519/jospt.2016.6311

17. Hengeveld E, Banks K, 1959-Maitland, G. D. G. D. P. m. Maitland's peripheral manipulation. 4th ed. Edinburgh; New York: Elsevier/Butterworth Heinemann; 2005.

18. Primeau C, et al. Trajectories of perceived exertion and pain over a 12-week neuromuscular exercise program in patients with knee osteoarthritis. Osteoarthr Cartil. 2020;28(11):1427-31. https://doi.org/10.1016/j.joca.2020.07.011.

19. Naugle KM, Fillingim RB, Riley $U$ 3rd. A meta-analytic review of the hypoalgesic effects of exercise. J Pain. 2012;13(12):1139-50. https://doi.org/1 0.1016/j.jpain.2012.09.006.

20. Hall M, Dobson F, Plinsinga M, Mailloux C, Starkey S, Smits E, et al. Effect of exercise on pain processing and motor output in people with knee osteoarthritis: a systematic review and meta-analysis. Osteoarthr Cartil. 2020; 28(12):1501-13. https://doi.org/10.1016/j.joca.2020.07.009.

21. Bandura A. Self-efficacy: toward a unifying theory of behavioral change. Psychol Rev. 1977;84(2):191-215. https://doi.org/10.1037//0033-295x.84.2.191.

22. Keefe FJ, Kashikar-Zuck S, Opiteck J, Hage E, Dalrymple L, Blumenthal JA. Pain in arthritis and musculoskeletal disorders: the role of coping skills training and exercise interventions. J Orthop Sports Phys Ther. 1996;24(4): 279-90. https://doi.org/10.2519/jospt.1996.24.4.279.

23. Hurley MV, Mitchell HL, Walsh N. In osteoarthritis, the psychosocial benefits of exercise are as important as physiological improvements. Exerc Sport Sci Rev. 2003;31(3):138-43. https://doi.org/10.1097/00003677-200307000-00007.
24. Cross M, Smith E, Hoy D, Nolte S, Ackerman I, Fransen M, et al. The global burden of hip and knee osteoarthritis: estimates from the global burden of disease 2010 study. Ann Rheum Dis. 2014;73(7):1323-30. https://doi.org/1 0.1136/annrheumdis-2013-204763.

25. Nuernberg Back CG, Liebano RE, Avila MA. Perspectives of implementing the biopsychosocial model to treat chronic musculoskeletal pain in primary health care. Pain Manag. 2021;11(2):217-25. https://doi.org/10.2217/pmt-202 0-0024.

26. Focht BC, Rejeski WJ, Ambrosius WT, Katula JA, Messier SP. Exercise, selfefficacy, and mobility performance in overweight and obese older adults with knee osteoarthritis. Arthritis Rheum. 2005;53(5):659-65. https://doi.org/1 0.1002 /art.21466.

27. Hott A, Brox Jl, Pripp AH, Juel NG, Liavaag S. Predictors of pain, function, and change in patellofemoral pain. Am J Sports Med. 2020;48(2):351-8. https://doi.org/10.1177/0363546519889623.

28. Hurley MV, Walsh N, Bhavnani V, Britten N, Stevenson F. Health beliefs before and after participation on an exercised-based rehabilitation programme for chronic knee pain: doing is believing. BMC Musculoskelet Disord. 2010;11. https://doi.org/10.1186/1471-2474-11-31.

29. Bandura A, Adams NE, Beyer J. Cognitive processes mediating behavioral change. J Pers Soc Psychol. 1977;35(3):125-39. https://doi.org/10.1037// 0022-3514.35.3.125.

30. Yilmaz H, Karaca G, Demir Polat HA, Akkurt HE. Comparison between depression levels of women with knee osteoarthritis, rheumatoid arthritis, and fibromyalgia syndrome: a controlled study. Turk J Phys Med Rehabil. 2015;61(3):197-202. https://doi.org/10.5152/tftrd.2015.87894.

31. Briani RV, et al. What interventions can improve quality of life or psychosocial factors of individuals with knee osteoarthritis? A systematic review with meta-analysis of primary outcomes from randomised controlled trials. Br J Sports Med. 2018;52:1031-+. https://doi.org/10.1136/bjsports-201 7-098099.

32. Hurley $M$, et al. Exercise interventions and patient beliefs for people with hip, knee or hip and knee osteoarthritis: a mixed methods review. Cochrane Database Syst Rev. 2018;4:Cd010842. https://doi.org/10.1002/14651858.CD01 0842.pub2.

33. Xie $\mathrm{SH}$, Wang $\mathrm{Q}$, Wang $L Q$, Wang $L$, Song KP, He CQ. Effect of internetbased rehabilitation programs on improvement of pain and physical function in patients with knee osteoarthritis: systematic review and metaanalysis of randomized controlled trials. J Med Internet Res. 2021;23(1): e21542. https://doi.org/10.2196/21542.

34. Chan A-W, et al. SPIRIT 2013 statement: defining standard protocol items for clinical trials. Ann Intern Med. 2013;158:200-+. https://doi.org/10.7326/ 0003-4819-158-3-201302050-00583.

35. Altman R, Asch E, Bloch D, Bole G, Borenstein D, Brandt $K$, et al. Development of criteria for the classification and reporting of osteoarthritis. Classification of osteoarthritis of the knee. Diagnostic and Therapeutic Criteria Committee of the American Rheumatism Association. Arthritis Rheum. 1986;29(8):1039-49. https://doi.org/10.1002/art.1780290816.

36. Altman RD, Hochberg M, Murphy WA Jr, Wolfe F, Lequesne M. Atlas of individual radiographic features in osteoarthritis. Osteoarthr Cartil. 1995; 3(Suppl A):3-70.

37. Adams R. Revised physical activity readiness questionnaire. Can Fam Physician. 1999:45:992-995, 1004-1005.

38. Tam E, Gandesbery BT, Young L, Borson S, Gorodeski EZ. Graphical instructions for administration and scoring the mini-cog: results of a randomized clinical trial. J Am Geriatr Soc. 2018;66(5):987-91. https://doi. org/10.1111/jgs.15313.

39. Bennell KL, Egerton T, Wrigley TV, Hodges PW, Hunt M, Roos EM, et al. Comparison of neuromuscular and quadriceps strengthening exercise in the treatment of varus malaligned knees with medial knee osteoarthritis: a randomised controlled trial protocol. BMC Musculoskelet Disord. 2011;12(1): 276. https://doi.org/10.1186/1471-2474-12-276.

40. Bennell KL, Kyriakides M, Metcalf B, Egerton T, Wrigley TV, Hodges PW, et al. Neuromuscular versus quadriceps strengthening exercise in patients with medial knee osteoarthritis and Varus Malalignment. Arthritis Rheumatol. 2014;66(4):950-9. https://doi.org/10.1002/art.38317.

41. Bennell KL, Nelligan RK, Kimp AJ, Schwartz S, Kasza J, Wrigley TV, et al. What type of exercise is most effective for people with knee osteoarthritis and comorbid obesity?: the TARGET randomized controlled trial. Osteoarthr Cartil. 2020;28(6):755-65. https://doi.org/10.1016/j.joca.2020.02.838. 
42. Hawker GA, Mian S, Kendzerska T, French M. Measures of adult pain: visual analog scale for pain (VAS pain), numeric rating scale for pain (NRS pain), McGill pain questionnaire (MPQ), short-form McGill pain questionnaire (SF$M P Q)$, chronic pain grade scale (CPGS), short form-36 bodily pain scale (SF$36 \mathrm{BPS})$, and measure of intermittent and constant osteoarthritis pain (ICOAP). Arthritis Care Res. 2011;63(Suppl 11):S240-52. https://doi.org/10.1 002/acr.20543.

43. Borg G, Ljunggren G, Ceci R. The increase of perceived exertion, aches and pain in the legs, heart rate and blood lactate during exercise on a bicycle ergometer. Eur J Appl Physiol Occup Physiol. 1985;54(4):343-9. https://doi. org/10.1007/bf02337176.

44. Hardware B, Anne Lacey E, Shewan J. Towards the development of a tool to assess educational needs in patients with arthritis. Clin Eff Nurs. 2004;8(2): 111-7. https://doi.org/10.1016/j.cein.2004.06.001.

45. French SD, Bennell KL, Nicolson PJA, Hodges PW, Dobson FL, Hinman RS. What do people with knee or hip osteoarthritis need to know? An international consensus list of essential statements for osteoarthritis. Arthritis Care Res. 2015;67(6):809-16. https://doi.org/10.1002/acr.22518.

46. Gay C, Chabaud A, Guilley E, Coudeyre E. Educating patients about the benefits of physical activity and exercise for their hip and knee osteoarthritis. Systematic literature review. Ann Phys Rehabil Med. 2016; 59(3):174-83. https://doi.org/10.1016/j.rehab.2016.02.005.

47. Kroon FP, et al. Self-management education programmes for osteoarthritis Cochrane Database Syst Rev. 2014:Cd008963. https://doi.org/10.1002/14651 858.CD008963.pub2.

48. Zhao $\mathrm{H}$, et al. Cross-cultural validation of the educational needs assessment tool into Chinese for use in severe knee osteoarthritis. Patient Prefe Adherence. 2018;12:695-705. https://doi.org/10.2147/ppa.S163492.

49. National Clinical Guideline Centre (UK). Osteoarthritis: Care and Management in Adults. London: National Institute for Health and Care Excellence (UK); 2014. PMID: 25340227.

50. Rausch Osthoff AK, Niedermann K, Braun J, Adams J, Brodin N, Dagfinrud H, et al. 2018 EULAR recommendations for physical activity in people with inflammatory arthritis and osteoarthritis. Ann Rheum Dis. 2018;77(9):125160. https://doi.org/10.1136/annrheumdis-2018-213585.

51. van Doormaal MCM, Meerhoff GA, Vliet Vlieland TPM, Peter WF. A clinical practice guideline for physical therapy in patients with hip or knee osteoarthritis. Musculoskelet Care. 2020. https://doi.org/10.1002/msc.1492.

52. Associationg, O. G. O. C. O. Guidelines for the diagnosis and treatment of osteoarthritis (2018). Chin J Orthop. 2018;38:705-15. https://doi.org/10.3760/ cma.j.issn.0253-2352.2018.12.001.

53. Wang X, Urban H, Bennell KL, Dickson C, Dobson F, Fransen M, et al. My joint pain, a web-based resource, effects on education and quality of care at 24 months. BMC Musculoskelet Disord. 2020;21(1):79. https://doi.org/10.11 86/s12891-020-3074-2

54. Bellamy N, Carette S, Ford PM, Kean WF, le Riche NG, Lussier A, et al. Osteoarthritis antirheumatic drug trials. II. Tables for calculating sample size for clinical trials. J Rheumatol. 1992;19(3):444-50.

55. Bellamy N, Buchanan WW, Goldsmith CH, Campbell J, Stitt LW. Validation study of WOMAC: a health status instrument for measuring clinically important patient relevant outcomes to antirheumatic drug therapy in patients with osteoarthritis of the hip or knee. J Rheumatol. 1988;15(12): 1833-40.

56. Tubach F, Ravaud P, Baron G, Falissard B, Logeart I, Bellamy N, et al. Evaluation of clinically relevant changes in patient reported outcomes in knee and hip osteoarthritis: the minimal clinically important improvement. Ann Rheum Dis. 2005;64(1):29-33. https://doi.org/10.1136/ard.2004.022905.

57. Burrows NJ, Booth J, Sturnieks DL, Barry BK. Acute resistance exercise and pressure pain sensitivity in knee osteoarthritis: a randomised crossover trial. Osteoarthr Cartil. 2014;22(3):407-14. https://doi.org/10.1016/j.joca.2013.12.023.

58. Bennell K, Dobson F, Hinman R. Measures of physical performance assessments: self-paced walk test (SPWT), stair climb test (SCT), six-minute walk test (6MWT), chair stand test (CST), timed up \& go (TUG), sock test, lift and carry test (LCT), and car task. Arthritis Care Res. 2011;63(Suppl 11):S35070. https://doi.org/10.1002/acr.20538.

59. Sullivan MJL, Bishop SR, Pivik J. The pain catastrophizing scale: development and validation. Psychol Assess. 1995;7(4):524-32. https://doi.org/10.1037/104 0-3590.7.4.524

60. Kori S, Miller R, Todd D. Kinisophobia: a new view of chronic pain behavior. Pain Manag. 1990;3:35-43.
61. Resnick B, Jenkins LS. Testing the reliability and validity of the self-efficacy for exercise scale. Nurs Res. 2000;49(3):154-9. https://doi.org/10.1097/000061 99-200005000-00007.

62. Campbell-Sills L, Stein MB. Psychometric analysis and refinement of the Connor-Davidson resilience scale (CD-RISC): validation of a 10-item measure of resilience. J Trauma Stress. 2007;20(6):1019-28. https://doi.org/10.1002/ jts.20271.

63. Kerns RD, Turk DC, Rudy TE. The West Haven-Yale multidimensional pain inventory (WHYMPI). Pain. 1985;23(4):345-56. https://doi.org/10.1016/0304-3 959(85)90004-1.

64. Den Oudsten BL, Van Heck GL, Van der Steeg AFW, Roukema JA, De Vries J. Personality predicts perceived availability of social support and satisfaction with social support in women with early stage breast cancer. Support Care Cancer. 2010;18(4):499-508. https://doi.org/10.1007/s00520-009-0714-3.

65. Helgeson VS. Social support and quality of life. Qual Life Res. 2003;12(Suppl 1):25-31. https://doi.org/10.1023/a:1023509117524.

66. Ware JE Jr, Sherbourne CD. The MOS 36-item short-form health survey (SF36). I. Conceptual framework and item selection. Med Care. 1992;30:473-83.

67. Sundararajan V, Henderson T, Perry C, Muggivan A, Quan H, Ghali WA. New ICD-10 version of the Charlson comorbidity index predicted in-hospital mortality. J Clin Epidemiol. 2004;57(12):1288-94. https://doi.org/10.1016/j. jclinepi.2004.03.012.

68. Taylor-Piliae RE, et al. Validation of a new brief physical activity survey among men and women aged 60-69 years. Am J Epidemiol. 2006;164:598606. https://doi.org/10.1093/aje/kwj248\%J.

69. Tennant R, Hiller L, Fishwick R, Platt S, Joseph S, Weich S, et al. The WarwickEdinburgh mental well-being scale (WEMWBS): development and UK validation. Health Qual Life Outcomes. 2007;5(1):63. https://doi.org/10.11 86/1477-7525-5-63.

70. Helen B, Robin P.Applied Mixed Models in Medicine, Second Edition, Chapter 6. New York: Wiley; 2006. p. 215-270. https://doi.org/10.1002/04 70023589

71. Honghu L, Tongtong W. Sample size calculation and power analysis of time-averaged difference. J Mod Appl Stat Methods. 2005;4:434-45.

72. Diggle PJ, Liang KY, Zeger SL. Analysis of longitudinal data. Chapter 2. New York: Oxford University Press; 1994.

73. Xie S-H, et al. The feasibility and effectiveness of internet-based rehabilitation for patients with knee osteoarthritis: a study protocol of randomized controlled trial in the community setting. Medicine. 2020;99. https://doi.org/10.1097/md.0000000000022961.

74. Schill W. Applied longitudinal data analysis for epidemiology: a practical guide. 2004;60(1):291-2. https://doi.org/10.1111/j.0006-341X.2004.172_7.x.

75. Liang K-Y, Zeger SL. Longitudinal data analysis using generalized linear models. Biometrika. 1986;73:13-22. https:/doi.org/10.1093/biomet/73.1.13\%J.

76. Krause MR, Serlin RC, Ward SE, Rony RY, Ezenwa MO, Naab F. Testing mediation in nursing research: beyond Baron and Kenny. Nurs Res. 2010; 59(4):288-94. https://doi.org/10.1097/NNR.0b013e3181dd26b3.

77. Baron RM, Kenny DA. The moderator-mediator variable distinction in social psychological research: conceptual, strategic, and statistical considerations. J Pers Soc Psychol. 1986;51(6):1173-82. https://doi.org/10.1037//0022-3514.51. 6.1173.

78. Farrell AD. Structural equation modeling with longitudinal data: strategies for examining group differences and reciprocal relationships. J Consult Clin Psychol. 1994;62(3):477-87. https://doi.org/10.1037//0022-006x.62.3.477.

79. Emsley R, Dunn G, White IR. Mediation and moderation of treatment effects in randomised controlled trials of complex interventions. Stat Methods Med Res. 2010;19(3):237-70. https://doi.org/10.1177/0962280209105014.

80. Enders C, Bandalos D. The relative performance of full information maximum likelihood estimation for missing data in structural equation models. Struc Equ Model. 2001;8. https://doi.org/10.1207/S1532 8007SEM0803_5.

81. Dell'Isola A, Jonsson T, Nero H, Eek F, Dahlberg L. Factors associated with the outcome of a first-line intervention for patients with hip or knee osteoarthritis or both: data from the BOA register. Phys Ther. 2020;100(10): 1771-81. https://doi.org/10.1093/ptj/pzaa113.

82. Deveza LA, Melo L, Yamato TP, Mills K, Ravi V, Hunter DJ. Knee osteoarthritis phenotypes and their relevance for outcomes: a systematic review. Osteoarthr Cartil. 2017;25(12):1926-41. https://doi.org/10.1016/j.joca.2017.08.009.

83. Runhaar J, Luijsterburg P, Dekker J, Bierma-Zeinstra SM. Identifying potential working mechanisms behind the positive effects of exercise therapy on 
pain and function in osteoarthritis; a systematic review. Osteoarthr Cartil. 2015;23(7):1071-82. https://doi.org/10.1016/j.joca.2014.12.027.

84. Hanley JA, Negassa A, Edwardes MD, Forrester JE. Statistical analysis of correlated data using generalized estimating equations: an orientation. Am J Epidemiol. 2003;157(4):364-75. https://doi.org/10.1093/aje/kwf215.

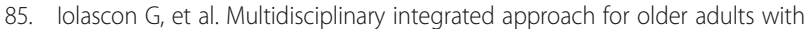
symptomatic osteoarthritis: SIMFER and SI-GUIDA Joint Position Statement. Eur J Phys Rehabil Med. 2020;56:112-9. https://doi.org/10.23736/s19739087.19.05837-4

86. Rice D, McNair P, Huysmans E, Letzen J, Finan P. Best evidence rehabilitation for chronic pain part 5: osteoarthritis. J Clin Med. 2019;8. https://doi.org/1 0.3390/jcm8111769.

87. Alschuler KN, Kratz AL, Ehde DM. Resilience and vulnerability in individuals with chronic pain and physical disability. Rehabil Psychol. 2016;61(1):7-18. https://doi.org/10.1037/rep0000055.

88. Thompson KA, Bulls HW, Sibille KT, Bartley EJ, Glover TL, Terry EL, et al. Optimism and psychological resilience are beneficially associated with measures of clinical and experimental pain in adults with or at risk for knee osteoarthritis. Clin J Pain. 2018;34(12):1164-72. https://doi.org/10.1097/ajp. 0000000000000642

\section{Publisher's Note}

Springer Nature remains neutral with regard to jurisdictional claims in published maps and institutional affiliations.

Ready to submit your research? Choose BMC and benefit from:

- fast, convenient online submission

- thorough peer review by experienced researchers in your field

- rapid publication on acceptance

- support for research data, including large and complex data types

- gold Open Access which fosters wider collaboration and increased citations

- maximum visibility for your research: over $100 \mathrm{M}$ website views per year

At BMC, research is always in progress.

Learn more biomedcentral.com/submissions 